\title{
Tunable and Polarization-Controlled High-Contrast Bright and Dark Coherent Resonances in Potassium
}

\author{
Silvia Gozzini ${ }^{1}$, Andrea Fioretti ${ }^{1}$, Alessandro lucchesini ${ }^{1}$, Luca Marmugi ${ }^{1,2,}$, Carmela \\ Marinelli ${ }^{1,3}$, Stoyan Tsvetkov ${ }^{4}$, Sanka Gateva ${ }^{4}$, and Stefka Cartaleva ${ }^{4}$ \\ ${ }^{1}$ Istituto Nazionale di Ottica, INO-CNR, SS Pisa, Via Moruzzi 1, 56124 Pisa, Italy. \\ ${ }^{2}$ Department of Physics and Astronomy, University College London, Gower Street, London WC1E 6BT, United Kingdom. \\ ${ }^{3}$ Department of Physical Sciences, Earth and Environment, Università di Siena, via Roma 56, 53100 Siena, Italy. \\ ${ }^{4}$ Institute of Electronics, Bulgarian Academy of Sciences, 72 Tzarigradsko Chaussee blvd., 1784 Sofia, Bulgaria. \\ ${ }^{*}$ Corresponding author: I.marmugi@ucl.ac.uk
}

Compiled July 24, 2017

This is a preprint version of the article appeared in Opt. Lett. 42, 15, 2930-2933 (2017) DOI: http:// doi.org/10.1364/ OL.42.002930. One print or electronic copy may be made for personal use only. Systematic reproduction and distribution, duplication of any material in this paper for a fee or for commercial purposes, or modifications of the content of this paper are prohibited.

We demonstrate high-contrast Electromagnetically Induced Absorption (EIA) bright resonances on the $D_{1}$ line of ${ }^{39} \mathrm{~K}$ with characteristics comparable to those of the Electromagnetically Induced Transparency (EIT) dark resonances observed in the same conditions. EIA is produced by the interaction of a weak probe beam with the atomic ground state driven in a degenerate coherent superposition by either a co- or counterpropagating pump beam. We have obtained an order of magnitude increase of the EIA's contrast with respect to previous similar experiments, performed with other alkalis, without compromising its linewidth. Furthermore, we show that the magneto-optic resonances can be continuously tuned from EIT to EIA by changing the relative handedness of circular polarizations of pump and probe beams, or depending on whether they co- or counter-propagate. This opens new perspectives in the use of EIA in a broad range of physical domains and in a large wealth of potential applications in optics and photonics.

OCIS codes: $\quad$ (020.1670), (020.3690), (020.7490)

https://doi.org/10.1364/OL.42.002930

Contrary to Coherent Population Trapping and Electromagnetically Induced Transparency (EIT) with their ultra-narrow linewidths [1-8], Electromagnetically Induced Absorption (EIA) has found limited applications, mainly due to its broader linewidth and significantly smaller contrast. Nonetheless, an increasing interest in EIA was recently generated in view of potential applications, such as enhancement of the group velocity of light [9] and photonic metamaterials [10].

EIA linewidths of several tens of kiloHertz have been reported, either with bichromatic excitation of Zeeman sublevels
[11] or in Hanle configuration [12-14]. Bi-chromatic Hanle configuration has been explored by using $R b D_{1}$ and $D_{2}$ lines [15]. Low-contrast EIA, resulting from EIT on $\mathrm{D}_{2}$ line, was reported. Higher contrast EIA has been observed in [16], although overlapped to large background, which could be detrimental for highly-selective applications. More recently, sub-kHz EIA resonances were observed in $\mathrm{Rb}[17]$ and $\mathrm{Cs}[18,19]$. In both cases, however, the contrast was of only a few percents. A pump/probe scheme in Hanle configuration for high-contrast EIA was proposed in a buffered vapor cell [20]. To date, however, no experimental demonstration has been reported. Highcontrast EIA has been observed on the ${ }^{87} \mathrm{Rb} \mathrm{D}_{2}$ line by using a complex excitation scheme with three separate photonic fields [21], and was theoretically investigated in [22]. EIA was also observed in the Paschen-Back regime in a purely non-degenerate three-level ladder system in ${ }^{87} \mathrm{Rb}$ [23]. Furthermore, while $\mathrm{Rb}$ and Cs have been widely investigated, this is the first observation of EIA in K, confirming our preliminary results [24].

In this Letter, we report on an alternative and simpler approach for large contrast EIA in Hanle configuration using a pump/probe excitation scheme. We have observed EIA resonance contrast exceeding $50 \%$ in thermal $\mathrm{K}$ vapor. In this case, hyperfine optical pumping, which accumulates atoms to levels non interacting with the light $[25,26]$, is largely compensated thanks to the substantial overlapping of the potassium $\mathrm{D}_{1}$ hyperfine transitions. Coherent resonances are produced by a low intensity probe beam interacting with the ground atomic state that has been driven in Hanle configuration by a pump laser beam. The sign of the coherent resonance (i.e. EIT or EIA) can be controlled by varying independently the circular polarization handedness of both laser beams. In particular, with counter-propagating beams, opposite circular polarizations produce EIT resonances, while circular polarization of the same handedness produce EIA resonances. The effect is opposite in the co-propagating configuration. Reversal of the coherent resonances has been previously reported with co-propagating beams and only partial accumulation of atoms in the bright state 
[16]. In our work, by taking advantage of the overlapping of the $\mathrm{K}$ ground state hyperfine components and of the optical transitions of its $\mathrm{D}_{1}$ line, we obtain the same efficiency of accumulation in states forming bright or dark resonances, depending on the experimental conditions, which can be easily tuned, as demonstrated in the following. These results represent a relevant advance in the EIA performances and relevant simplification of the setup (see, for example, $[15,21]$ ). Also, thanks to the possibility of using counter-propagating beams, new experimental protocols and arrangements can be thus envisaged, as well as new possible applications in optics and photonics.

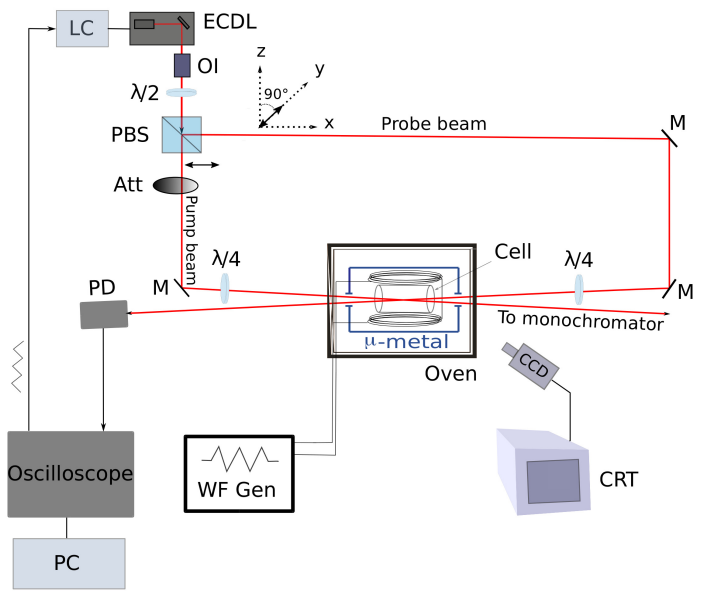

Fig. 1. Counter-propagating configuration. ECDL: extended cavity diode laser; LC: laser control; OI: optical insulator; PBS: Polarizing beam splitter; M: Mirror; Att: variable neutral density filter; WF Gen: waveform generator; PD: Si photodiode.

The experimental setup is shown in Fig. 1, in the configuration with counter-propagating beams. In the co-propagating arrangements the two beams are overlapped and propagate collinearly. A natural mixture of ${ }^{39} \mathrm{~K}(93.3 \%)$ and ${ }^{41} \mathrm{~K}(6.7 \%)$ is contained in a polydimethylsiloxane (PDMS)-coated Pyrex cell. The organic coating prevents spin relaxation upon atom/wall collisions $[26,27]$. We recall here that the K hyperfine (hf) ground (excited) state splitting, $461.7 \mathrm{MHz}(55.5 \mathrm{MHz})$, is smaller than the Doppler width, $773.4 \mathrm{MHz}$ at $300 \mathrm{~K}$. Consequently, the optical hyperfine transitions are not resolved and we observe in the co-propagating scheme a single Gaussian-like absorption profile [28], while in the counter-propagating scheme the profile is modified by saturated absorption (Fig. 2).

An extended cavity diode laser (ECDL, Toptica DL 100) is tuned to the $\mathrm{D}_{1}$ line of ${ }^{39} \mathrm{~K}(770.1 \mathrm{~nm})$. The beam is split in two counter- (or co-) propagating pump (up to $5 \mathrm{~mW}$ ) and probe (between 10 and $50 \mu \mathrm{W}$ ) beams. The two beams are then overlapped along the cell main axis with a waist of $5 \mathrm{~mm}$. The maximum intensity used in this work is around $6 \mathrm{~mW} / \mathrm{cm}^{2}$, corresponding to a ratio $\mathrm{I}_{L} / \mathrm{I}_{S} \approx 3.5$ where $\mathrm{I}_{S}=1.71 \mathrm{~mW} / \mathrm{cm}^{2}$ is the saturation intensity of $\mathrm{KD}_{1}$ line [28]. The intensities as well as the polarizations of the two beams can be independently controlled by neutral density filters and waveplates. The vapor cell is placed in a single layer $\mu$-metal cylinder. Inside, a pair of Helmholtz coils provides a uniform transverse magnetic field scanned across zero. The $\mathrm{K}$ density is controlled by means of a resistive heater (not shown in Fig. 1). The probe absorption is measured by a Si photodiode and acquired as a voltage signal by a digital oscilloscope. EIT/EIA resonances are observed on this beam at zero transverse magnetic field, in degenerate conditions, in the so-called Hanle configuration [29].

In a first set of experiments, both the laser frequency and the magnetic field are swept across their resonant values. The laser frequency is scanned across the $\mathrm{D}_{1}$ line resonance by modulating the voltage supplied to the piezoelectric stack controlling the ECDL diffraction grating, with a $5 \mathrm{~Hz}$ triangular waveform. At the same time, the magnetic field is modulated by a second triangular waveform at $200 \mathrm{~Hz}$, phase-locked to the previous one. The field is continuously swept in the typical interval $B=0 \pm 500 \mathrm{mG}$, where $B=0$ is the zero field condition where Hanle EIT/EIA is observed. In a second set of experiments, the magnetic field is scanned around $\mathrm{B}=0$ and the laser is kept at a constant frequency (typically, at the maximum of the EIT or the EIA resonance), to measure the absolute characteristics of the EIT and EIA resonances. Both experiments are repeated in co- and counter-propagating configurations with different combinations of polarizations.

In Fig. 2 we show the EIT/EIA conversion on the K absorption profile with counter-propagating beams. The typical spectral structures due to saturated absorption are visible [30]. Each time the magnetic field is $\mathrm{B}=0$, a narrow coherent resonance is observed. The Hanle resonance is produced at degenerate conditions when the laser beams couples Zeeman sublevels of the ground states $\left(\mathrm{F}_{g}=1,2\right)$ and the excited states $\left(\mathrm{F}_{e}=1,2\right)$.

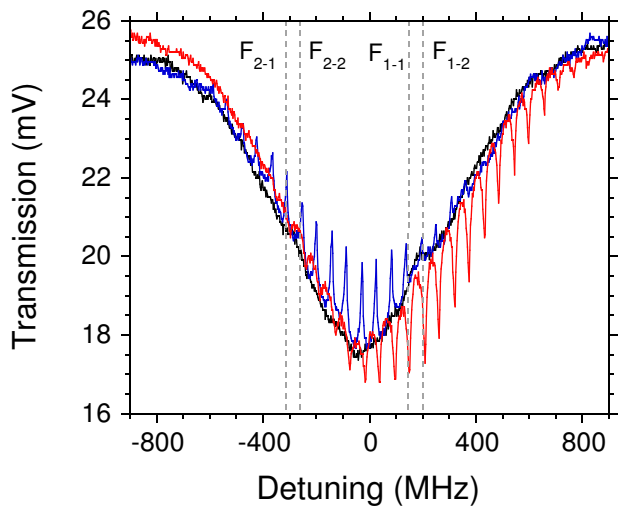

Fig. 2. Probe transmission in counter-propagating configuration: EIT (blue curve, upward peaks) conversion to EIA (red curve, downward peaks); spectroscopy profile (black curve). Dashed vertical lines mark the hyperfine optical transitions $\mathrm{F}_{g}$ $\rightarrow \mathrm{F}_{e} . \mathrm{T}=50.0^{\circ} \mathrm{C} ; \mathrm{P}_{\text {pump }}=2.91 \mathrm{~mW} ; \mathrm{P}_{\text {probe }}=30 \mu \mathrm{W}$.

In Fig. 2, EIA resonances appear only in the "blue" side of the absorption profile. This is consistent with the fact that this resonance is observed for transitions $\mathrm{F}_{g} \rightarrow \mathrm{F}_{e}=\mathrm{F}_{g}+1[12,13]$. On the contrary, EIT is formed by transitions $\mathrm{F}_{g} \rightarrow \mathrm{F}_{e}=\mathrm{F}_{g}$ or $\mathrm{F}_{e}=\mathrm{F}_{g}-1$. Here, the nature of the resonance (i.e. EIT or EIA) depends on the handedness of the circular polarizations of probe and pump beams, once their mutual alignment is fixed. The results are summarized in Tab. 1 and Fig. 3. The EIA formation mechanism is unique to the $\mathrm{K}$ hyperfine structure, thus leading to an enhanced efficiency of accumulation in the bright state, superior or comparable to those obtained with more complex schemes and experimental setups, in thermal vapors [15, 21]. 
Table 1. Conversion of EIT to EIA: results overview.

\begin{tabular}{cccc}
\hline Configuration & Handedness & Resonance & Ref. \\
\hline Co-propagating & Equal & EIT & Fig. 3.a) \\
Co-propagating & Opposite & EIA & Fig. 3.b) \\
Counter-propagating & Equal & EIA & Fig. 3.c) \\
Counter-propagating & Opposite & EIT & Fig. 3.d) \\
\hline
\end{tabular}

In detail, co-propagating pump and probe beams with the same circular polarization produce EIT: both photon fields act in the same direction to accumulate atoms in a non-absorbing state at $\mathrm{B}=0$ as observed in standard Hanle configuration (Fig. 3.a)). In the case of opposite circular polarizations, the intense pump beam accumulates atoms in the most absorbing state examined by the probe beam, thus producing enhanced absorption or EIA (Fig. 3.b)).

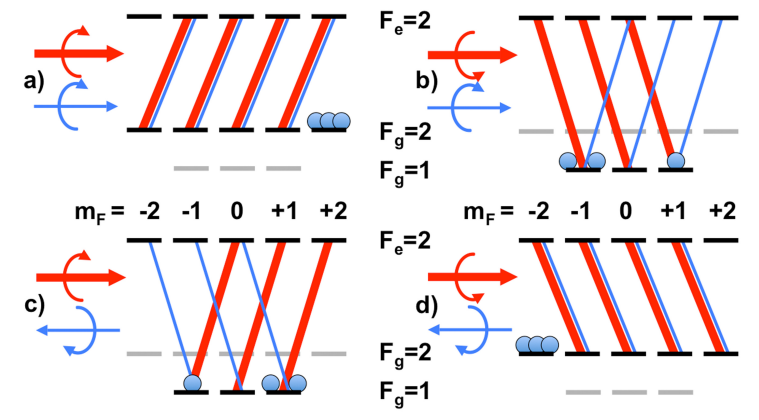

Fig. 3. Conversion from EIT (a) to EIA (b) in the copropagating and from EIA (c) to EIT (d) in the counterpropagating configurations. Only the optical transitions $\mathrm{F}_{g}=1,2 \rightarrow \mathrm{F}_{e}=2$ are shown. Analogous explanations apply to the transitions $\mathrm{F}_{g}=1,2 \rightarrow \mathrm{F}_{e}=1$, which produce only EIT.

The effect is opposite in the counter-propagating case, where opposite polarizations produce EIT resonances (Fig. 3.d)). This is consistent with [31], where a longitudinal magnetic field was used. On the contrary, when counter-propagating pump and probe beams have the same circular polarization, the interaction produces an EIA peak (Fig. 3.c)). When the same circular polarization, here defined according to its handedness and by choosing $\hat{y}$ as the quantization axis, is given to probe and pump, $\mathrm{K}$ interacts with photons carrying opposite $\mathrm{L}_{z}= \pm \hbar$ quanta. Effectively, atoms interact with $\sigma^{+}$light from one beam and $\sigma^{-}$ photons from the other. As a result, the two beams which previously cooperated in the creation of a dark state are now pushing atoms towards opposite $\mathrm{m}_{F}$ states, thus producing EIA. Incidentally, we note that the spin polarizations of the dark states created by EIT are opposite in the counter-propagating and copropagating cases, namely $\left|m_{F}=+2\right\rangle$ and $\left|m_{F}=-2\right\rangle$ (Figs. 3.a) and d)). This could have a relevant impact for state manipulation in atomic physics.

The EIT/EIA peak profiles are acquired at the laser frequency maximizing the amplitude and then fitted with Lorentzian curves to extract information on amplitude, contrast and linewidth. We have measured a Full Width at Half Maximum (FWHM) of $40.29 \pm 0.03 \mathrm{kHz}$ in the best EIT case, while EIA has a FWHM=66.4 $\pm 0.6 \mathrm{kHz}$ (Fig. 4).

Figure 4 shows that the maximum efficiency of the coherent

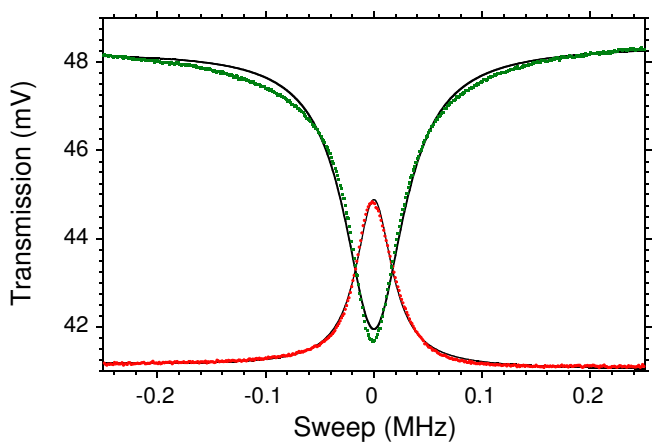

Fig. 4. EIA (top) and EIT (bottom) line profiles. Green squares (downward curve): experimental EIA data; black thick line: Lorentzian fit; red dots (upward curve): experimental EIT data; black thin line: Lorentzian fit. $\mathrm{T}=50{ }^{\circ} \mathrm{C}, \mathrm{I}_{L}=0.4 \mathrm{~mW} / \mathrm{cm}^{2}$, copropagating configuration.

resonances can be obtained by re-tuning the laser: the transmitted light level is different for EIT (whose maximum is typically observed at the center of the absorption profile) and EIA (whose maximum is instead observed around the frequency of the $\mathrm{F}_{g}=1 \rightarrow \mathrm{F}_{e}=2$ transition).

In addition, the FWHM has been found to be fitted by a square root function of the laser intensity (Fig. 5). The agreement with the simple fit suggests that the main contribution to this broadening is power broadening (see, among others, [32]). This indirectly confirms the role of both photonic fields in the creation of coherent resonances.

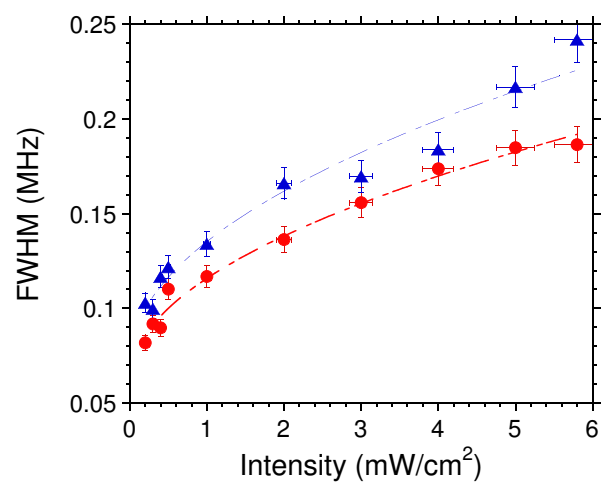

Fig. 5. Resonance FWHM versus pump beam intensity in counter-propagating configuration. Blue triangles: EIA; Red circles: EIT.

In Fig. 6.a) we show the amplitude A of the coherent resonances as function of the pump beam intensity. EIT and EIA exhibit very similar heights. This leads to a remarkable EIA/EIT $\approx 1$ ratio. The resonance contrast, defined as $\mathrm{C}=\mathrm{A} /\left(\left|\mathrm{I}_{\text {absorbed }}\right|\right)$, is instead larger for EIA (Fig. 6.b), due to the fact that the best coherent resonance, contrary to the EIT one, is detuned from the maximum of absorption.

In conclusion, we have demonstrated a new experimental approach for EIA with narrow linewidth and unprecedented contrast. The system is based on a pump/probe scheme in Hanle configuration realized in $\mathrm{K}$ vapor in a PDMS-coated cell. We have obtained EIA resonances with amplitude comparable with 

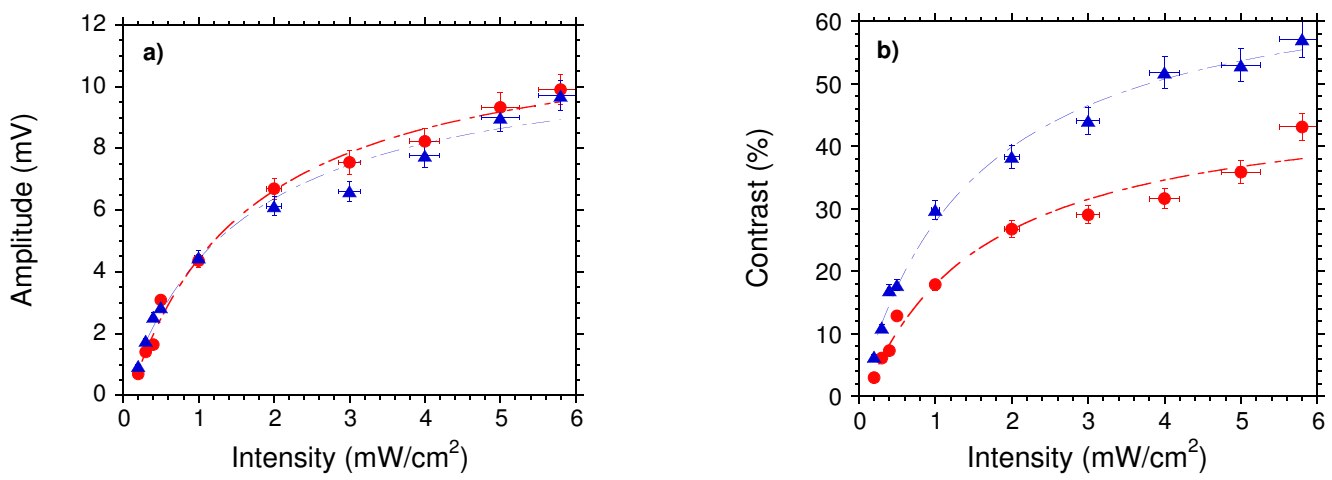

Fig. 6. Resonance amplitude a) and contrast b) versus pump beam intensity in counter-propagating configuration. Blue triangles: EIA; Red circles: EIT.

that of EIT and more than 1.5 larger contrast. FWHMs as low as $66 \mathrm{kHz}$ were obtained (1.65 times broader than the corresponding EIT). Furthermore, by taking advantage of the K hyperfine structure aiding efficient transfer between the two ground state levels, clear tunability of the atomic response was obtained. This allows easy, potentially fast and continuous switching from EIT to EIA and intermediate configurations, with different degrees of opacity, controlled by handedness of pump and probe and/or by their mutual orientation.

Our results thus disclose new paths for EIA applications, from state manipulation in fundamental physics and metrology, to tunable and narrowband optical and photonic devices. Examples include: atomic optical isolators [33, 34], atom-based polarization filters and splitters, atomic filters with sub-MHz band [35-37], and cavity-less bistable systems and switches [38, 39], all with potential for reduced dissipation, fast switching time and efficiency tunability, not to mention control of light group velocity [9] and novel photonic metamaterials [10].

$7^{\text {th }}$ EU Framework Programme "COSMA" (PIRSES-GA-2012295264). Italian National Research Council and Bulgarian Academy of Sciences (CNR/BAS 2013-2015). National Science Fund of Bulgaria (DN 08-19 /14.12.2016).

\section{REFERENCES}

1. J. Vanier, Applied Physics B 81, 421 (2005).

2. M. Erhard and H. Helm, Phys. Rev. A 63, 043813 (2001).

3. J. Belfi, G. Bevilacqua, V. Biancalana, S. Cartaleva, Y. Dancheva, K. Khanbekyan, and L. Moi, J. Opt. Soc. Am. B 26, 910 (2009).

4. V. I. Yudin, A. V. Taichenachev, Y. O. Dudin, V. L. Velichansky, A. S. Zibrov, and S. A. Zibrov, Phys. Rev. A 82, 033807 (2010).

5. R. Wynands and A. Nagel, Applied Physics B 68, 1 (1999).

6. C. Ottaviani, D. Vitali, M. Artoni, F. Cataliotti, and P. Tombesi, Phys. Rev. Lett. 90, 197902 (2003).

7. L. V. Hau, S. E. Harris, Z. Dutton, and C. H. Behroozi, Nature 397, 594 (1999).

8. A. H. Safavi-Naeini, T. P. M. Alegre, J. Chan, M. Eichenfield, M. Winger, Q. Lin, J. T. Hill, D. E. Chang, and O. Painter, Nature 472, 69 (2011).

9. Y.-S. Lee, H. J. Lee, and H. S. Moon, Opt. Express 21, 22464 (2013).

10. X. Zhang, N. Xu, K. Qu, Z. Tian, R. Singh, J. Han, G. S. Agarwal, and W. Zhang, Scientific Reports 5, 10737 EP (2015).

11. A. M. Akulshin, S. Barreiro, and A. Lezama, Phys. Rev. A 57, 2996 (1998).

12. F. Renzoni, S. Cartaleva, G. Alzetta, and E. Arimondo, Phys. Rev. A 63, 065401 (2001).

13. F. Renzoni, C. Zimmermann, P. Verkerk, and E. Arimondo, Journal of Optics B: Quantum and Semiclassical Optics 3, S7 (2001).
14. Y. Dancheva, G. Alzetta, S. Cartaleva, M. Taslakov, and C. Andreeva, Optics Communications 178, 103 (2000).

15. A. A. Zhukov, S. A. Zibrov, G. V. Romanov, Y. O. Dudin, V. V. Vassiliev, V. L. Velichansky, and V. P. Yakovlev, Phys. Rev. A 80, 033830 (2009).

16. N. Ram, and M. Pattabiraman, J. Phys. B 43, 24, 245503 (2010).

17. H.-J. Kim and H. S. Moon, Opt. Express 19, 168 (2011).

18. S. R. Chanu, K. Pandey, and V. Natarajan, Europhys. Lett. 98, 4 (2012).

19. H. Ravi, M. Bhattarai, V. Bharti and V. Natarajan, Europhys. Lett. 117, 6 (2017).

20. D. V. Brazhnikov, A. V. Taichenachev, A. M. Tumaikin, and V. I. Yudin, Laser Physics Letters 11, 125702 (2014).

21. I. Ben-Aroya, and G. Eisenstein, Opt. Express 19, 10, 9956 (2011).

22. R. S. Grewal and M. Pattabiraman, J. Phys. B: At. Mol. Opt. Phys. 47, 195501 (2014).

23. D. J. Whiting, E. Bimbard, J. Keaveney, M. A. Zentile, C. S. Adams, and I. G. Hughes, Opt. Lett. 40, 18, 4289-4292 (2015).

24. S. Gozzini, A. Lucchesini, C. Marinelli, L. Marmugi, S. Gateva, S. Tsvetkov, and S. Cartaleva, Journal of Physics: Conference Series 700, 012051 (2016).

25. S. Gozzini, S. Cartaleva, A. Lucchesini, C. Marinelli, L. Marmugi, D. Slavov, and T. Karaulanov, Eur. Phys. J. D 53, 153 (2009).

26. K. Nasyrov, S. Gozzini, A. Lucchesini, C. Marinelli, S. Gateva, S. Cartaleva, and L. Marmugi, Phys. Rev. A 92, 043803 (2015).

27. M. V. Balabas, T. Karaulanov, M. P. Ledbetter, and D. Budker, Phys. Rev. Lett. 105, 070801 (2010).

28. R. K. Hanley, P. D. Gregory, I. G. Hughes, and S. L. Cornish, Journal of Physics B: Atomic, Molecular and Optical Physics 48, 195004 (2015).

29. F. Renzoni, W. Maichen, L. Windholz, and E. Arimondo, Phys. Rev. A 55, 3710 (1997).

30. D. Bloch, M. Ducloy, N. Senkov, V. Velichansky, and V. Yudin, Las. Phys. 6, 670 (1996).

31. M. Klein, M. Hohensee, D. F. Phillips, and R. L. Walsworth, Phys. Rev. A 83, 013826 (2011).

32. M. L. Citron, H. R. Gray, C. W. Gabel, and C. R. Stroud, Phys. Rev. A 16, 1507 (1977)

33. L. Weller, K. S. Kleinbach, M. A. Zentile, S. Knappe, I. G. Hughes, and C. S. Adams, Opt. Lett. 37, 3405 (2012).

34. C. Sayrin, C. Junge, R. Mitsch, B. Albrecht, D. O'Shea, P. Schneeweiss, J. Volz, and A. Rauschenbeutel, Phys. Rev. X 5, 041036 (2015).

35. J. A. Zielińska, F. A. Beduini, N. Godbout, and M. W. Mitchell, Opt. Lett. 37, 524 (2012).

36. X. Zhao, X. Sun, M. Zhu, X. Wang, C. Ye, and X. Zhou, Opt. Express 23, 17988 (2015).

37. M. A. Zentile, D. J. Whiting, J. Keaveney, C. S. Adams, and I. G. Hughes, Opt. Lett. 40, 2000 (2015).

38. D. X. Khoa, L. V. Doai, L. N. M. Anh, L. C. Trung, P. V. Thuan, N. T. Dung, and N. H. Bang, J. Opt. Soc. Am. B 33, 735 (2016).

39. P. Kumar and S. Dasgupta, Phys. Rev. A 94, 023851 (2016). 